\title{
Estimating Epidemic Parameters: Application to H1N1 Pandemic Data
}

\author{
Elissa J. Schwartz ${ }^{\mathrm{a}}$, Boseung Choi ${ }^{\mathrm{b}}$, Grzegorz A. Rempala ${ }^{\mathrm{c}, *}$ \\ ${ }^{a}$ School of Biological Sciences and Department of Mathematics, Washington State \\ University, Pullman WA 99164, USA \\ ${ }^{b}$ Department of Computer Science and Statistics, Daegu University, Gyeongbuk 712-714, \\ Republic of Korea \\ ${ }^{c}$ Division of Biostatistics and Mathematical Biosciences Institute, The Ohio State \\ University, Columbus, OH 43210, USA
}

\begin{abstract}
This paper discusses estimation of the parameters in an SIR epidemic model from the observed longitudinal new infection count data. The potential problems of the standard MLE approaches are revealed and possible remedies suggested. The analysis is based on the epidemic data from the 2009 outbreak of H1N1 influenza on the campus of Washington State University.

Keywords: H1N1 influenza, stochastic SIR model, parameter estimation, Monte Carlo approximation

2010 MSC: 92D30, 92-08, 62M-05
\end{abstract}

\section{Introduction}

The model of an infectious disease spread in a homogenous closed population (one without birth, death or migration) is know historically as the SIR model of Kermack and McKendrick (1927). It divides the population into three groups: susceptibles, infectives, and removed. In order to describe the epidemic's dynamics, modern SIR models typically use the formalism of Markov processes. Such Markovian models have the advantage of simplicity, as they can be easily simulated; however, they are not always simple

\footnotetext{
* Corresponding author.

Email addresses: ejs@wsu.edu (Elissa J. Schwartz), bchoi@daegu.ac.kr (Boseung Choi), rempala.3@osu.edu (Grzegorz A. Rempala)
}

Preprint submitted to Mathematical Biosciences

March 20, 2015

C) 2015. This manuscript version is made available under the Elsevier user license http://www.elsevier.com/open-access/userlicense/1.0/ 
to fit to empirical data, partially because the empirical datasets are rarely obtained from closed systems. One of the major metrics of the SIR model is its basic reproduction number $\left(R_{0}\right)$, that is, the average number of secondary infections contracted from a single infected individual. The current paper looks at some issues surrounding the estimation of $R_{0}$, and more generally, fitting the classical Markov SIR model to a closed epidemic dataset. As our data example, we have chosen the data from the 2009 H1N1 outbreak at Washington State University.

The Fall 2009 outbreak of H1N1 influenza (commonly known as swine $\mathrm{flu}$ ) on the campus of Washington State University (WSU) was one of many episodes of the global swine flu pandemic that rolled over the United States and the world after its initial outbreak in the state of Veracruz, Mexico in April 2009. The illness was determined to be caused by a new strain of H1N1 virus, $\mathrm{A}(\mathrm{H} 1 \mathrm{~N} 1) \mathrm{pdm09}$, a result of a previous triple reassortment of bird, swine and human flu viruses further combined with a Eurasian pig flu virus, leading to the term "swine" flu (Trifonov et al., 2009). Unlike most strains of influenza, A(H1N1)pdm09 does not disproportionately infect adults older than 60 years, and spreads easily among young, healthy adults. In 2009, the outbreaks on college campuses were quite frequent, although overall, the timing of the pandemic was fortunate, with its peak intensity largely falling on the college summer break period of May-August in the continental US. The WSU outbreak at its Pullman, WA campus was likely initiated immediately upon the student body return for Fall 2009 classes in late August 2009. By the time it was over, almost 2,300 students had come down with flu-like symptoms that, being outside the usual time window for seasonal flu outbreaks, were associated with the H1N1 virus. (For the first ten days of the outbreak, all suspected cases were tested and laboratoryconfirmed to be H1N1, after which all cases were considered H1N1.) The analysis of this particular pandemic dataset is interesting, due to the unique geography of the WSU Pullman campus, located in a typical college town with a large student population (around 18,000) and a relatively small local one (around 9,000) in a somewhat remote part of the state of Washington (the average population density in the county outside the city of Pullman is only about 8 households per square mile). Consequently, given a relatively limited inflow and outflow of individuals during an academic year, the WSU dataset may be considered as obtained from an approximately closed (population) system, which greatly facilitates its analysis. 


\subsection{WSU Dataset}

The original dataset consists of the daily counts of the new infections on the WSU campus for the period from August to December 2009, as reported to the campus student health services. The counts were obtained directly from the cases of "influenza-like illness" among students who visited or called the WSU student health services seeking care. The data collection started on August 22, 2009 when the first group of 11 students was seen by the clinic. For the purpose of our statistical analysis, 101 reported daily counts were considered as records of "new infectives". To obtain the total daily count of infectives, these counts were combined with the estimated number of previously infected who did not yet recover. The recovery was assumed to follow the Markov process outlined in the next section with the average period of recovery being 5 days, based on some earlier estimates (Choi and Rempala, 2012). The resulting daily counts of infectives are plotted for reference in Figure 1. The data may have been subject to both over-reporting and under-reporting, as described in Schwartz et al. (2014), as some students may have incorrectly presumed they had H1N1 when they had other influenzalike illnesses, while other students may have decided not to seek medical care. Overall though misreporting was considered as a relatively minor issue for the dataset (Schwartz et al., 2014). The total student body population at the onset of an epidemic was taken as 18,234. Before we proceed to analyze the dataset depicted in Figure 1, for the benefits of the readers less familiar with the underlying statistical theory, we provide the necessary basic facts in the next section. For more detailed introduction, see, e.g., the monograph by Andersson and Britton (2000). For another study analyzing the WSU dataset, see also Vaidya et al. (2014).

\section{Markovian Epidemic Models}

The simplest stochastic epidemic model may be viewed as a stochastic dynamical system of, say, $u$ individual types (e.g., $u=3$ for SIR with susceptible, infective, or removed) evolving according to some continuous time Markov jump process. The state of the epidemic at time $t$ is an $l$-vector $\mathcal{X}(t)$ giving the number of individuals (or units) of each type. We assume a finite number of jump (transition) types, with the $k$-th type determined by a vector of inputs $\nu_{k}$ and outputs $\nu_{k}^{\prime}$ specifying, respectively, the number of units of each type consumed and created in the $k$-th transition. The transition rate 


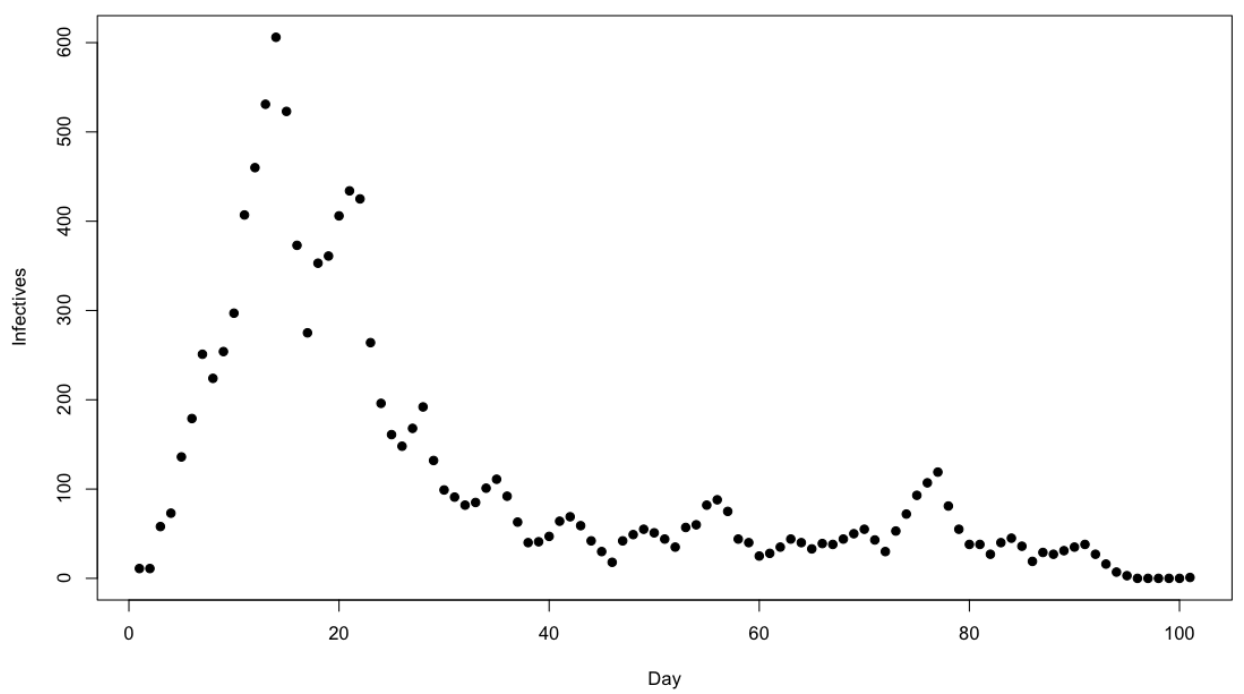

Figure 1: Swine Flu Epidemic Data. H1N1 daily infective counts on the Pullman campus of WSU for the period of 101 days in Fall 2009, as in Miller et al. (2013).

is given by the function $h_{k}(x)$. Specifically, if the interaction occurs at time $t$, the new state becomes

$$
\mathcal{X}(t)=\mathcal{X}(t-)+\nu_{k}^{\prime}-\nu_{k}
$$

and the number of times that the $k$-th transition occurs by time $t$ is given by the counting process satisfying

$$
N_{k}(t)=\mathcal{Y}_{k}\left(\int_{0}^{t} h_{k}(X(s)) d s\right)
$$

where the $\mathcal{Y}_{k}$ are independent unit Poisson processes. Note that writing $N_{k}$ in this form makes it clear why $h_{k}$ is referred to as a rate, since it is indeed the rate of a corresponding Poisson process. The state of the system $X(t)$ satisfies the following trajectory equation (see, e.g., Andersson and Britton, 2000, Chapter 5)

$$
\begin{aligned}
\mathcal{X}(t) & =\mathcal{X}(0)+\sum_{k} N_{k}(t)\left(\nu_{k}^{\prime}-\nu_{k}\right) \\
& =\mathcal{X}(0)+\left(\nu^{\prime}-\nu\right) N(t)
\end{aligned}
$$

where $\nu^{\prime}$ is the matrix with columns given by the $\nu_{k}^{\prime}, \nu$ is the matrix with columns given by the $\nu_{k}$, and $N(t)$ is the (column) vector with components 
$N_{k}(t)$. Recent attempts to model chemical reactions taking place in biological cells, as well as to analyze early stages of epidemics (O'Neill and Roberts, 1999; Ball et al., 2006), have led to renewed interest in stochastic models following (2) (see, e.g., Choi and Rempala 2012). The rate $h_{k}(t)$ is typically assumed to be proportional to the number of distinct subsets of those units present at time $t$ that can form inputs for the $k$-th transition. Intuitively, this assumption reflects the idea that the population of units of interest forms a well-stirred closed physical system, and is commonly referred to in the chemical physics and demographics literature as the law of mass action (LMA) (see, e.g., Turner et al., 2004). Formally, denoting $\left|\nu_{k}\right|=\sum_{i} \nu_{i k}$, LMA assumes that the rate function has the form

$$
h_{k}(x, \theta)=\theta_{k} \frac{\prod_{i} \nu_{i k} !}{n^{\left|\nu_{k}\right|-1}}\left(\begin{array}{c}
x \\
\nu_{1 k} \cdots \nu_{u k}
\end{array}\right)=n \theta_{k} \frac{\prod_{i} \nu_{i k} !\left(\begin{array}{c}
x_{i} \\
\nu_{i k}
\end{array}\right)}{n^{\left|\nu_{k}\right|}},
$$

where $n$ is an "effective volume" parameter proportional to the total stochastic system size (i.e., the combined count of all existing units). The vector parameter $\theta$ above consists of the values $\theta_{k}$ for $k \geq 1$, which are reactionspecific (in general, interaction-specific) kinetic constants. It is assumed that the vector $\theta_{k}(k \geq 1)$, along with the initial state vector $\left(\mathcal{X}(0)=\theta_{0}\right)$, fully parametrize the system (2).

Denoting by $c=n^{-1} x$ the vector of unit concentrations, and considering the behavior of the mass action rate functions as $n \rightarrow \infty$, one obtains

$$
h_{k}(x, \theta) \approx n \theta_{k} \prod c_{i}^{\nu_{i k}}=: n \theta_{k} \tilde{h}_{k}(c) .
$$

This approximation, along with the Poisson law of large numbers, which states that for a Poisson process $Y$ and large $n, Y(n u) / n \approx u$, yield the following deterministic (ODE) system, which approximates, for large $n$, the stochastic system (2):

$$
\dot{c}(t)=\sum_{k} \theta_{k} \tilde{h}_{k}(c)\left(\nu_{k}^{\prime}-\nu_{k}\right)
$$

As indicated above, the LMA tacitly assumes that the populations of interest are both spatially and phenotypically homogeneous, i.e., they constitute a well-stirred physical system (see, e.g., Wilkinson, 2009). Whereas these assumptions are not expected to be satisfied in many realistic scenarios, in the case of the WSU dataset, they are expected to hold pretty well. 
For some related discussion, see e.g., (Mills et al., 2004; Balcan et al., 2009). As a footnote to this section's discussion, let us also remark that it is often possible to reduce a non-homogenous system to a homogenous one via the compartmentalization method, the technique that is routinely used for modeling stochastic spatial features of complex biological models (Gibson and Renshaw, 1998) and also in the context of spatial epidemics (see, e.g., Hohle et al. (2005), Streftaris and Gibson (2004) for within-herd dynamics of disease, or recently Kouyos et al. (2010) for changes in spatial HIV transmissions in Switzerland).

\section{Complete Likelihood}

For the $\theta_{k}(k \geq 1)$ parameters, the likelihood function of interest may be derived by considering the density of the process (2) evaluated at its complete trajectory. To this end, denote the complete process trajectory by $\boldsymbol{X}=\{\mathcal{X}(t)\}_{t=0}^{T}$ where both the timings and types of all interactions between the species in the network up to a fixed time point $T<\infty$ are assumed to be known. For the process with $v$ interactions, denote by $r_{k}$ the number of interactions of type $k, k=1,2, \ldots, v$ in the time interval $(0, T]$, with $r=\sum_{k} r_{k}$, and denote by $t_{i}$ and $k_{i}$, respectively, the time and type of the $i$-th occurring interaction. To construct the likelihood function, we consider the (conditional) transition density function for the $i$ th event, which, under our Markov model (in view of the well-known result about arrivals from two independent exponential variables), consists of an independent pair of the event's time and type. This representation leads to the popular "direct" Gillespie algorithm (Gibson and Bruck, 2000) for simulating such Markov models. The complete likelihood function for $\theta_{k}$ on $(0, T]$ may be written as (cf., e.g., Boys et al., 2008)

$$
L(\theta \mid \boldsymbol{X})=\prod_{i=1}^{r} h_{k_{i}}\left(x\left(t_{i-1}\right), \theta_{k_{i}}\right) \exp \left(\int_{0}^{T} h_{0}(x(t), \theta) d t\right)
$$

where $h_{0}(x(t), \theta)=\sum_{k=1}^{v} h_{k}\left(x(t), \theta_{k}\right)$. By the LMA (4), for fixed $n$,

$$
h_{k}\left(x, \theta_{k}\right)=\theta_{k} g_{k}(x) \quad k=1 \ldots, v
$$


where $g_{k}(\cdot)$ is a rational function free of $\theta$, we note a useful factorization of the likelihood (6)

$$
\begin{aligned}
\mathcal{L}(\theta \mid \boldsymbol{X}) & =\left(\prod_{i=1}^{r} \theta_{k_{i}} g_{k_{i}}\left(x\left(t_{i}\right)\right)\right) \exp \left(-\int_{0}^{T} \sum_{k=1}^{v} \theta_{k} g_{k}(x(t)) d t\right) \\
& \propto \prod_{k=1}^{v} \theta_{k}^{r_{k}} \exp \left(-\theta_{k} \int_{0}^{T} g_{k}(x(t)) d t\right) \\
& =\prod_{k=1}^{v} \mathcal{L}_{k}\left(\theta_{k} \mid \boldsymbol{X}\right),
\end{aligned}
$$

where the last equation defines quantities $\mathcal{L}_{k}\left(\theta_{k} \mid \boldsymbol{X}\right)$. It follows that the likelihood inference may be carried out for each $\theta_{k}$ separately, by maximizing the corresponding $\mathcal{L}_{k}\left(\theta_{k} \mid \boldsymbol{X}\right)$ quantity which is proportionate to the gamma density function $\Gamma\left(r_{k}+1, \int_{0}^{T} g_{k}(x(t))\right)$. By differentiating with respect to each $\theta_{k}$, we obtain their unique maximum likelihood estimates (MLEs) at the modes of the corresponding marginal gamma densities

$$
\hat{\theta}_{k}=\frac{\sum_{i=1}^{T} r_{k i}}{\int_{0}^{T} g_{k}(x(t)) d t}=\frac{r_{k}}{\int_{0}^{T} g_{k}(x(t)) d t}, \quad k=1, \ldots, v .
$$

Note that each $\hat{\theta}_{k}$ may be also interpreted as the method of moments estimator, i.e., the solution of the estimating equation

$$
\int_{0}^{T} d N_{k}(t)-\theta_{k} \int_{0}^{T} g_{k}(x(t)) d t=0, \quad k=1, \ldots, v
$$

where $N_{k}(t)$ is the $k$-th interaction counting process (1) (see, e.g., Andersson and Britton 2000 Chapter 9, for more details).

The Bayesian analysis in the above setting is also quite straightforward. Due to the form of the likelihood function (8), a family of independent gamma distributions arrives naturally as a set of conjugate priors, that is, $\theta_{k} \sim$ $\Gamma\left(a_{k}, b_{k}\right), k=1, \ldots, v$. Under this family of priors, the application of Bayes' theorem produces posterior gamma distributions that retain independence, i.e., for $k=1, \ldots, v$ we have

$$
\theta_{s} \mid \boldsymbol{x} \sim \Gamma\left(a_{k}+r_{k}, b_{k}+\int_{0}^{T} g_{k}(x(t)) d t\right), \quad k=1, \ldots, v .
$$


In view of this, the maximum aposteriori (MAP) estimator is simply the mode of this posterior distribution, i.e., an adjusted MLE. In particular, in case of the uninformative and improper priors with $a_{k}=1$ and $b_{k}=0$, MLE and MAP estimator coincide. For more details on the Bayesian approach, see Boys et al. (2008).

\section{Fitting SIR Model to WSU Dataset}

\subsection{MLE Analysis}

Since the classical SIR epidemic model of Kermack and McKendrick (1927) assumes that we have the set of three species: susceptibles $(X)$, infectives $(Y)$ and removed $(Z)$, with the initial conditions $X(0)=n$, and $Y(0)=m=\mu n$, the trajectory equation (2) with $\mathcal{X}(t)=(X(t), Y(t), Z(t))$ specializes to

$$
\left(\begin{array}{l}
X(t) \\
Y(t) \\
Z(t)
\end{array}\right)=\left(\begin{array}{c}
n \\
m \\
0
\end{array}\right)+N_{1}(t)\left(\begin{array}{c}
-1 \\
1 \\
0
\end{array}\right)+N_{2}(t)\left(\begin{array}{c}
0 \\
-1 \\
1
\end{array}\right)
$$

with

$$
\begin{aligned}
& N_{1}(t)=Y_{1}\left(\lambda \int_{0}^{t} X(s) Y(s) d s / n\right), \\
& N_{2}(t)=Y_{2}\left(\gamma \int_{0}^{t} Y(s) d s\right)
\end{aligned}
$$

according to (3). In the above model, $\lambda$ is the transmission rate parameter and $\gamma$ is the recovery rate parameter. Their respective MLE estimates (9) are

$$
\begin{aligned}
& \hat{\lambda}=\frac{n N_{1}(T)}{\int_{0}^{T} X(s) Y(s) d s} \\
& \hat{\gamma}=\frac{N_{2}(T)}{\int_{0}^{T} Y(s) d s} .
\end{aligned}
$$

Note that a more natural parameter to estimate than $\gamma$ is $1 / \gamma$ - the average length of infectious period - which may be estimated by $1 / \hat{\gamma}$. The estimates (11) are consistent in the sense that they converge to their respective population rates with probability one as $n \rightarrow \infty$. This is sometimes known in the 


\begin{tabular}{ccc}
\hline Parameter & MLE Value & SE \\
\hline$\lambda$ & 0.228 & 0.004 \\
$\gamma^{-1}$ & 5.235 & 0.111 \\
$R_{0}$ & 1.193 & 0.035 \\
\hline
\end{tabular}

Table 1: The numerical results of MLEs (11) and (12) for the H1N1 dataset.

literature as the large volume consistency (Rempala, 2012). In this sense, the consistent estimates of the standard errors for the above MLEs are, respectively, $S E(\hat{\lambda})=\hat{\lambda} / \sqrt{N_{1}(T)}$ and $S E\left(\hat{\gamma}^{-1}\right)=\hat{\gamma}^{-1} / \sqrt{N_{2}(T)}$. Similarly, the basic reproduction number $R_{0}=\lambda / \gamma$ is consistently estimated by its MLE

$$
\hat{R}_{0}=\hat{\lambda} / \hat{\gamma}
$$

It can be shown (see, e.g., Rida 1991) that as $n \rightarrow \infty$ the estimate $\hat{R}_{0}$ is asymptotically normal with mean $R_{0}$ and limiting standard deviation

$$
S E\left(\hat{R}_{0}\right)=\hat{R}_{0} \sqrt{\left(\bar{Z}^{-1}+(\bar{Z}+\mu)^{-1}\right) / n},
$$

where $\bar{Z}$ is the total fraction of removed: $\bar{Z}=1-X(T) / n$.

Assuming $\mu$ known and the closed epidemic model $N_{2}(T)=N_{1}(T)+\mu n$, note that

$$
X(s)=n-Y(s)-Z(s)+\mu n
$$

and therefore, the denominators in (11) may be estimated from the epidemic trajectory data by the trapezoidal rule, $\int_{0}^{t} f(s) d s \approx \sum_{i=0}^{t-1} f(i)+(f(t)-$ $f(0)) / 2$, valid for any continuous function $f:[0, t] \rightarrow \mathbb{R}$ and a sufficiently fine partition of the $[0, t]$ interval (e.g., daily counts in WSU dataset). This allows us to approximately compute $\hat{R}_{0}$ from (11) and (12).

The results of the MLE analysis for the H1N1 data in Figure 1 are presented in Table 1 . Note that since we modeled the recovery process based on the average duration of 5 days, the true value of $1 / \gamma$ is 5 and its MLE in Table 1 is approximately within 2SE of that value. This indicates that the MLE bias is not a major concern for this particular dataset. Overall, the results in Table 1 look deceptively (!) promising. We discuss this issue next.

\subsection{Problems with MLE}

Despite the fact that the MLE values in Table 1 seem reasonable at first glance, the attempt to validate the MLE-fitted model by comparing it to the 

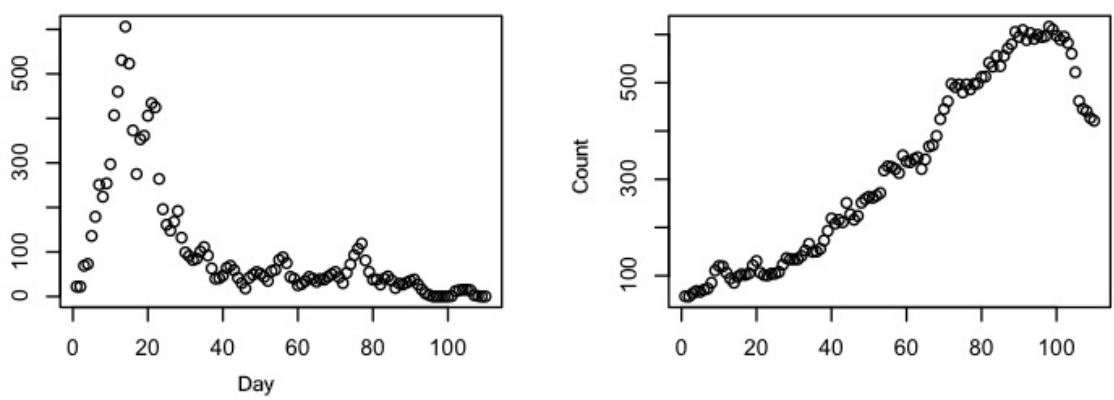

Figure 2: MLE Lack of Fit. Left panel: The daily infective counts from Figure 1 used to obtain the MLE values in Table 1. Right Panel: Daily counts generated from a typical realization of the Markov SIR model (10) with the same initial conditions and the parameter values from Table 1. The lack of agreement in the epidemic's dynamics for the two cases is clearly visible.

data fails dramatically, as depicted in Figure 2. The reason for this failure is not at first obvious but after a careful consideration of Figure 2 becomes quite apparent. The trajectories of the fitted MLE Markov epidemic model (right panel) have different dynamics than the original trajectory due to the fact that the original number of reported infectives $(m=11)$ at onset, apparently, cannot produce the pattern of a high peak of infections in the early days of the epidemic timeline (around day 15). Instead, this number of infectives is seen to result in a pattern of the right panel, where the peak occurs much later (around day 95). This is clearly not consistent with the data and indicates that the initial reported value of $m$ may be too low. Unfortunately, the MLE estimates tend to be very sensitive to the mis-specifcation of the initial infectives, as the denominators in (9) may depend on $m$ in a non-linear way. This is exactly the case for $\hat{\lambda}$ in (11). One standard way to remedy the problem of MLE onset bias is to consider a likelihood profiling approach, that is, to incorporate $m$ as a parameter into the MLE procedure. Unfortunately, this is typically difficult to accomplish directly as the formulae (9) depend on $m$ only implicitly. Consequently, the profiling method requires multiple reestimation of the parameters from data augmented with different values of $m$, along with subsequent verification of the results via model-data comparisons similar to the one in Figure 2. Needless to say, the efficiency of such a procedure may be low, and in general, its accuracy or precision cannot be guaranteed without additional assumptions. Fortunately, a simpler solution is available in the setting considered here. We discuss this point next. 


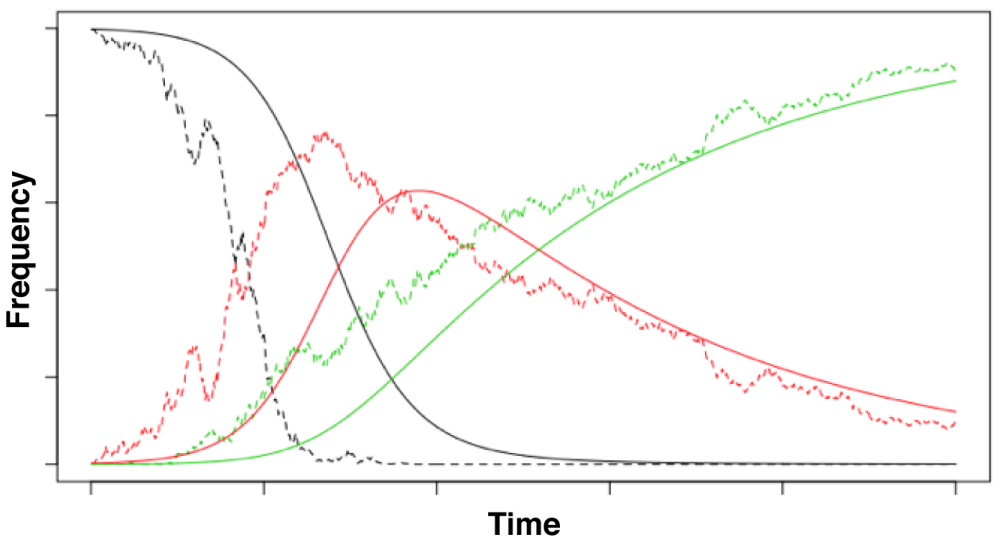

Figure 3: Deterministic vs. Stochastic SIR Model. An example of a scaled stochastic set of SIR trajectories (dashed) compared against the deterministic ones (solid) in the classical Kermack-McKendrick SIR model. The initial conditions and the rate values are identical for both cases $(\mu=0.005, \lambda=1 / 4, \gamma=1 / 5)$.

\subsection{Alternative Approach: Least Squares Estimation (LSE)}

Historically, the least squares method has been a popular way of fitting ODE models to trajectory data in a variety of settings, including many biological ones (e.g., Gani and Leach, 2001). Typically, the LSE method is applied under the assumption that the data is a result of the deterministic ODE trajectory observed with distortion caused by the additive (Gaussian) extrinsic noise (e.g., Cintrón-Arias et al., 2009). This is different from our present setting where we assume that the ODE (5) is a result of the averaged intrinsic (possibly non-Gaussian) noise of the stochastic trajectories of $\mathcal{X}(t)$ as $n \rightarrow \infty$. An immediate consequence of this difference is the fact that although the numerical values of the LSE estimates are the same under both noise models, their respective standard errors have very different forms (see, e.g., Wilkinson, 2009). It also follows that asymptotically (as $n \rightarrow \infty$ ), the distribution of the intrinsic noise LSE agrees (conditionally on the initial infectives) with that of the MLE given by (11).

In order to describe the fitting of the H1N1 dataset via LSE, we first need to introduce some additional notation. Assume that the collection of $l>1$ trajectory points $\left\{X\left(t_{i}\right)\right\}_{i=0}^{l}$ with $t_{0}=0$ is observed, where possibly some parts of the $X\left(t_{i}\right)$ may be missing at various $i$ 's.

For $i=1 \ldots, l$ let $c^{\theta}\left(t_{i}\right)$ be the deterministic limit (5) of $\overline{\mathcal{X}}\left(t_{i}\right)=\mathcal{X}\left(t_{i}\right) / n$ as $n \rightarrow \infty$. Assuming that $c^{\theta}(\cdot)$ is differentiable w.r.t. $\theta \in \Theta$, one may obtain 


\begin{tabular}{ccc}
\hline Parameter & LSE Value & SE \\
\hline$m$ & 105.1 & 40.45 \\
$\lambda$ & 0.24 & 0.02 \\
$\gamma^{-1}$ & 5.10 & 0.136 \\
$R_{0}$ & 1.228 & 0.039 \\
\hline
\end{tabular}

Table 2: The numerical results of LSE estimation for the H1N1 dataset.

the LSE estimate $\hat{\theta}$ as any solution of the equation

$$
\hat{\theta}=\operatorname{argmin}_{\theta \in \Theta} \sum_{i}\left\|\overline{\mathcal{X}}\left(t_{i}\right)-c^{\theta}\left(t_{i}\right)\right\|^{2} .
$$

The large volume consistency of the above LSE under mild conditions, such as trajectory identifiability, was recently discussed, for instance, in Rempala (2012) in the context of chemical models, but the results derived there apply to our present setting. In particular, from the central limit theorem derived in Rempala (2012), it follows that the Monte-Carlo approximations of the SEs for the LSE estimates are valid for sufficiently large population size $n$. This gives a convenient way of ascertaining the overall model fit to the observed trajectory data.

Recall that $m=\mu n$ for our SIR model discussed in Section 4.1, as well as $\theta=(\mu, \lambda, \gamma)$, with ODE (5) taking the form of an original KermackMcKendrick ODE for susceptible $(x)$, infectious $(y)$, and removed $(z)$ (see Kermack and McKendrick 1927). Therefore $c^{\theta}(t)=(x(t), y(t), z(t))$ is the solution of

$$
\begin{aligned}
\dot{x}(t) & =-\lambda x(t) y(t) \\
\dot{y}(t) & =\lambda x(t) y(t)-\gamma y(t) \\
\dot{z}(t) & =\gamma y(t)
\end{aligned}
$$

$x(0)=1$ and $y(0)=\mu<1, z(0)=0$. This is the basic classical model for epidemic disease spread within a fixed size population, with initial concentration $(1+\mu)$. For illustration, an example of its ODE trajectory against the corresponding stochastic one given by (10) is presented in Figure 3 for the parameters $(\mu=0.005, \lambda=1 / 4, \gamma=1 / 5)$ and $n=500$ (for the stochastic trajectory).

The results of the LSE analysis for the H1N1 dataset from Figure 1 are summarized in Table 2 and presented in Figure 4, where the model fit to 


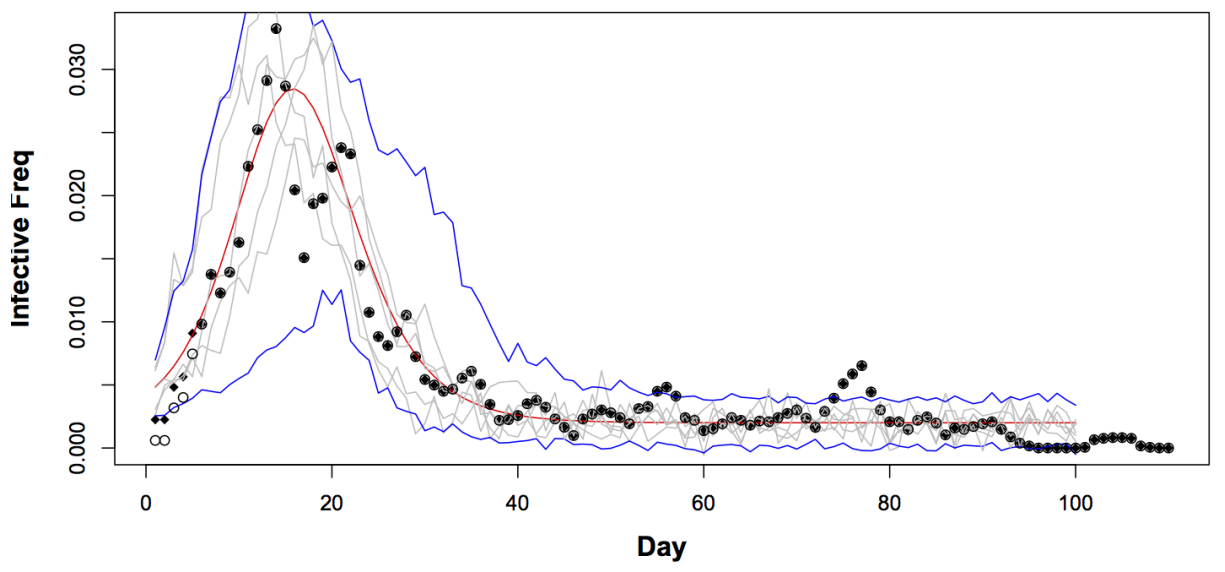

Figure 4: LSE Model Fit. The circles represent data from Figure 1 scaled by the population size, with open circles representing data points adjusted by new estimated $m$ value from Table 2 . The corresponding adjusted values are plotted as diamonds. The adjusted data for the most part fall within the approximate $95 \%$ confidence envelope for the stochastic model (10) which is the area between top and bottom curves. These confidence bounds are obtained by Monte-Carlo simulations of multiple stochastic trajectories of (10) (5 of which are shown in grey for illustration) which fluctuate around the deterministic SIR trajectory (13) represented by a smooth middle curve (red). The model fitting is truncated on day 101 so the final epidemic phase (after day 90) is not well-captured.

the scaled data (that is, with initial conditions $x(0)=1$ and $y(0)=\mu=$ $m / n, z(0)=0)$ is illustrated. Inspecting the entries in Table 2 , one notes that in addition to a much larger estimated number of initial infectives $(m=105$ vs. $m=11$ in the original dataset), the biggest difference between the values in Tables 1 and 2 is the 5 -fold increase in the standard error for the $\lambda$ parameter. There is also a relatively small but pronounced increase in the estimated value of $R_{0}$. Under-reporting by initial infectives could have been due to individuals who contracted H1N1 before arriving on campus (but were still infectious) who did not need to visit WSU student health services or who visited a health center prior to arrival. Altogether though, except for the number of initial infectives $m$, the numerical values for the remaining model parameters obtained using the LSE method do not seem to be markedly different from those obtained via MLE. Nevertheless, beyond the initial discrepancy, the LSE fitted model is seen to generally agree with 
the original trajectory data in Figure 1. This is illustrated in Figure 4, where the smooth middle curve represents the fitted ODE trajectory with parameter values from Table 2, and the top and bottom curves denote the $95 \%$ confidence envelope for the corresponding stochastic Markov model, for which several trajectories are also plotted. As seen from the plots, the data points in general fall within the confidence envelopes of the LSE fitted model. Note that the analysis is truncated at day 101 (due to the decreased data reliability beyond that time-point) and therefore the fitted model is biased in the final phase of the epidemic with its trajectory (for instance, its trajectory does not decrease to 0 as would be expected for any non-endemic outbreak).

\section{Summary and Discussion}

In this paper we looked at the problem of fitting a closed SIR model to observational data, using as an example a dataset from the 2009 H1N1 outbreak on the campus of Washington State University. Due to the relatively remote geographical location of the WSU campus, the dataset may be considered as representing an approximately closed epidemic. However, the standard approach based on the MLE failed to produce a model fit (specifically, the average number of secondary infections $R_{0}$ ) consistent with the observed process. It seems that the main reason for the poor fit was the fact that the standard MLE analysis is always conditional on the observed initial epidemic values. However, such values are often downward biased due to data underreporting. This results in a biased $R_{0}$ estimate, and, more importantly, often leads to the incorrect model trajectory dynamics. One way to correct for the onset bias is via a standard likelihood profiling method where the conditional likelihoods under different onset conditions are compared and the appropriate maximizer is selected. Computationally, the profiling and similar methods may be implemented via the Bayesian MCMC approach, but this may often prove cumbersome. Instead, we proposed here a conceptually simpler and less computer-intensive approach relying on the LSE method, where the initial epidemic conditions were fitted to the observed trajectory along with other model parameters. The approach was effective for the particular dataset considered, although the bias reduction came at the expense of increased variance of the parameter estimates. The increase was not too severe though, and overall the LSE analysis was able to fare much better then the standard MLE in recovering both the more realistic values of the model parameters (in particular $R_{0}$ ) and the reasonable pattern of dynamics 
consistent with the data.

\section{Acknowledgement}

The research was partially funded by the US National Science Foundation under grant DMS1318886 and by the National Research Foundation of Korea under grant 2012R1A1A1010156. The collaboration between the authors was also possible due to the generous support from the Mathematical Biosciences Institute at the Ohio State University where two of the authors were long-term visitors during the Fall 2011 program "Stochastics in Biological Systems".

The authors would like to thank the associate editor and two referees for their comments on an earlier version of the manuscript.

\section{References}

Andersson, H., Britton, T., 2000. Stochastic epidemic models and their statistical analysis. Springer Verlag.

Balcan, D., Hu, H., Goncalves, B., Bajardi, P., Poletto, C., Ramasco, J., Paolotti, D., Perra, N., Tizzoni, M., Broeck, W., et al., 2009. Seasonal transmission potential and activity peaks of the new influenza A (H1N1): a Monte Carlo likelihood analysis based on human mobility. BMC medicine $7(1), 45$.

Ball, K., Kurtz, T., Popovic, L., Rempala, G., 2006. Asymptotic analysis of multiscale approximations to reaction networks. Annals of Applied Probability 16 (4), 1925-1961.

Boys, R., Wilkinson, D., Kirkwood, T., 2008. Bayesian inference for a discretely observed stochastic kinetic model. Statistics and Computing 18 (2), $125-135$.

Choi, B., Rempala, G. A., Jan 2012. Inference for discretely observed stochastic kinetic networks with applications to epidemic modeling. Biostatistics 13 (1), 153-65.

Cintrón-Arias, A., Castillo-Chávez, C., Bettencourt, L. M., Lloyd, A. L., Banks, H., 2009. The estimation of the effective reproductive number from disease outbreak data. Math Biosci Eng 6 (2), 261-282. 
Gani, R., Leach, S., 2001. Transmission potential of smallpox in contemporary populations. Nature 414 (6865), 748-751.

Gibson, G., Renshaw, E., 1998. Estimating parameters in stochastic compartmental models using markov chain methods. Mathematical Medicine and Biology 15 (1), 19.

Gibson, M., Bruck, J., 2000. Efficient exact stochastic simulation of chemical systems with many species and many channels. J. Phys. Chem. A 104 (9), 1876-1889.

Hohle, M., Jorgensen, E., O’Neill, P., 2005. Inference in disease transmission experiments by using stochastic epidemic models. Journal of the Royal Statistical Society Series C-Applied Statistics 54, 349-366.

Kermack, W. O., McKendrick, A. G., 1991. Contributions to the mathematical theory of epidemics-I. (Reprint from the 1927 original). Bull Math Biol 53 (1-2), 33-55.

Kouyos, R. D., von Wyl, V., Yerly, S., Böni, J., Taffé, P., Shah, C., Bürgisser, P., Klimkait, T., Weber, R., Hirschel, B., Cavassini, M., Furrer, H., Battegay, M., Vernazza, P. L., Bernasconi, E., Rickenbach, M., Ledergerber, B., Bonhoeffer, S., Günthard, H. F., May 2010. Molecular epidemiology reveals long-term changes in HIV type 1 subtype B transmission in Switzerland. J Infect Dis 201 (10), 1488-97.

Miller, L., Jones, T., Morgan, M., Lapin, S., Schwartz, E., 2013. Individualbased computational model used to explain 2009 pandemic H1N1 in rural campus community. Journal of Biological Systems 21 (4), 1340005.

Mills, C., Robins, J., Lipsitch, M., Dec. 2004. Transmissibility of 1918 pandemic influenza. Nature 432 (7019), 904-906.

O’Neill, P., Roberts, G., 1999. Bayesian inference for partially observed stochastic epidemics. Journal of the Royal Statistical Society Series AStatistics In Society 162, 121-129.

Rempala, G. A., Aug 2012. Least squares estimation in stochastic biochemical networks. Bull Math Biol 74 (8), 1938-55. 
Rida, W. N., 1991. Asymptotic properties of some estimators for the infection rate in the general stochastic epidemic model. Journal of the Royal Statistical Society. Series B (Methodological), 269-283.

Schwartz EJ, Morgan M, Lapin S. Pandemic 2009 H1N1 influenza in two settings in a small community: the workplace and the university campus. Epidemiol Infect., in press. doi: 10.1017/S0950268814002684.

Streftaris, G., Gibson, G., Apr. 2004. Bayesian inference for stochastic epidemics in closed populations. Statistical Modelling 4 (1), 63-75.

Trifonov, V., Khiabanian, H., Rabadan, R., Jul 2009. Geographic dependence, surveillance, and origins of the 2009 influenza a (h1n1) virus. N Engl J Med 361 (2), 115-9.

Turner, T., Schnell, S., Burrage, K., 2004. Stochastic approaches for modelling in vivo reactions. Computational Biology and Chemistry 28 (3), 165178.

Vaidya NK, Morgan M, Jones T, Miller L, Lapin S, Schwartz EJ. Modelling the epidemic spread of an H1N1 influenza outbreak in a rural university town. Epidemiol Infect., in press. doi: 10.1017/S0950268814002568.

Wilkinson, D., 2009. Stochastic modelling for quantitative description of heterogeneous biological systems. Nature Reviews Genetics 10 (2), 122-133. 